\title{
Straight running stability of automobiles - experiments with a driving simulator
}

April 13, 2019

\author{
*Politecnico di Milano, Milan, Italy ** Danisi Engineering, Nichelino, Italy
}

\begin{abstract}
The stability of the straight ahead running motion of automobiles is studied with proper theoretical tools pertaining to bifurcation theory. The study is both theoretical and experimental. Four theoretical models have been employed. They refer respectively to one simple car model, one simple driver model, one complex car model and one complex driver model. The existence of bifurcations, namely Hopf bifurcations, is found for both the simple car/simple driver model combination and for the complex car/complex driver model combination. The experimental study refers to the employment of a driving simulator in which a human driver controls the complex car model. At the driving simulator, bifurcations are found which correspond to the ones predicted either with the simple car/simple driver model combination or the complex car/complex driver model combination. The correspondence is approximate, due mainly to the fact that especially the simple driver model seems inadequate to capture the actual human driver behaviour. After a subcritical Hopf bifurcation, a chaotic motion is found at the driving simulator. The driving simulator does not seem introducing factors affecting sensibly bifurcations, so the bifurcations that have been found appear to be real, i.e. bifurcations could be found on an actual car running straight ahead.
\end{abstract}

\section{Introduction}

Every common driver feels that a car, running straight ahead, may become unstable if a disturbance of sufficient level occurs. To describe accurately such an intuitive fact, an in-depth mathematical reasoning is needed. Nonlinear models of cars and nonlinear models of drivers are the only mathematical representations of reality that may work $[27,24,28]$. Often, classical academic books deal with the stability of cars referring to linearized models [10, 25]. This implies that the disturbance is limited. In other words, classical academic hypotheses on straight running stability of cars cannot provide any prediction if the disturbance is strong. Bifurcation theory covers this gap, as shown in $[27,8,6,5,7,18,26,34]$. To date, a sound experimental substantiation of bifurcation theory is lacking referring to straight running stability of cars. This paper aims at covering this gap, focusing on both mathematical modeling and experimental substantiation.

In the literature, a number of papers have been written on bifurcations of road vehicle models $[8,3,13,15,17,22,30,31,35,36]$. In [27] a pioneering early representation of phase portraits is given. Still today it seems that the current understanding of bifurcations would refer to simple vehicle models only, namely single-track or slightly more complex models. An analysis seems lacking on bifurcations occurring for complex vehicle models. The paper aims at covering this gap. We will not mention here the bifurcations of truck-trailer combination or car-trailer combination, because this would be out of scope. 
Referring to driver models, we refer to the main contributions available in the literature [28, $12,21,4]$ which deal with both linear and nonlinear models. Such contributions are fundamental to establish the basic understanding of how drivers act and form the basis for this paper. Driver models with delay seem necessary to produce bifurcations and to describe properly car driver behaviour $[33,19,20,18,6,5]$. In these papers bifurcation of vehicle and driver models refer usually to simple vehicle models. Such simple models have been preferably used due to the difficult usage of phase portraits referring to models with tens of state variables. Bifurcations of complex car models in combination with driver models seem not studied accurately in the literature. The paper aims at covering this gap.

Studying straight ahead running stability in the real world is quite dangerous. It is complicated to apply a significant disturbance to the vehicle and, especially at high speeds, loosing control leads directly to life threatening. So a driving simulator seems the only viable way to study running stability [29, 14]. Obviously, driving simulator is a complex machine that may introduce into the loop some damping or latence effects that might influence the accuracy and even the correctness of results. In big driving simulators, latence is an issue which implies enormous power demand. We used a mid-size driving simulator which has a mean latence of less than $20 \mathrm{~ms}$, a very low time lag that, as a first approximation, can be neglected with respect to driver's delay [2]. Since the driving simulator that has been employed does not seem introducing relevant factors that could influence the nature of bifurcation, we have decided to use it as a reliable means to study straight ahead running stability.

The paper is organized as follows. At first the four theoretical models are described. Then the bifurcations occurring during straight ahead motion are studied and compared. Then the Driving simulator is introduced and the results of the tests that have been performed are summarized and duly commented.

\section{System models}

To perform an accurate bifurcation analysis of the car \& driver system, a proper mathematical modelling has to be done.

\subsection{Simple car model}

Let us focus on the 2 degrees of freedom single-track model shown in Fi. 1(a). The degrees of freedom are the lateral motion and the yaw rotation. The longitudinal motion is not considered as a degree of freedom because the longitudinal speed is considered constant. Longitudinal forces $F_{x_{i}}$ are needed to keep constant the longitudinal speed, their magnitude is considered small and they are neglected. The lateral axle forces $F_{y_{i}}$ can be modelled by using the well known Pacejka Magic Formula [27] and refer, for this single-track model, to the whole axle characteristic

$$
F_{y_{i}}=D_{i} \sin \left(C_{i} \arctan \left(B_{i} \alpha_{i}-E_{i}\left(B_{i} \alpha_{i}-\arctan \left(B_{i} \alpha_{i}\right)\right)\right)\right)
$$

where $\alpha_{i}, i=\{f, r\}$ are the front and rear slip angles, defined as

$$
\alpha_{f}=\delta-\left(\frac{v+r a}{u}\right), \quad \alpha_{r}=-\left(\frac{v-r b}{u}\right) .
$$

where $a$ and $b$ are the distances from the centre of gravity of the front and rear axles, respectively. The coefficients of the Magic Formula define the shape of the axle characteristic and the handling properties of the vehicle. In this work two different configurations of axle characteristics have been used, one referring to an understeering vehicle and one referring to an oversteering vehicle. 


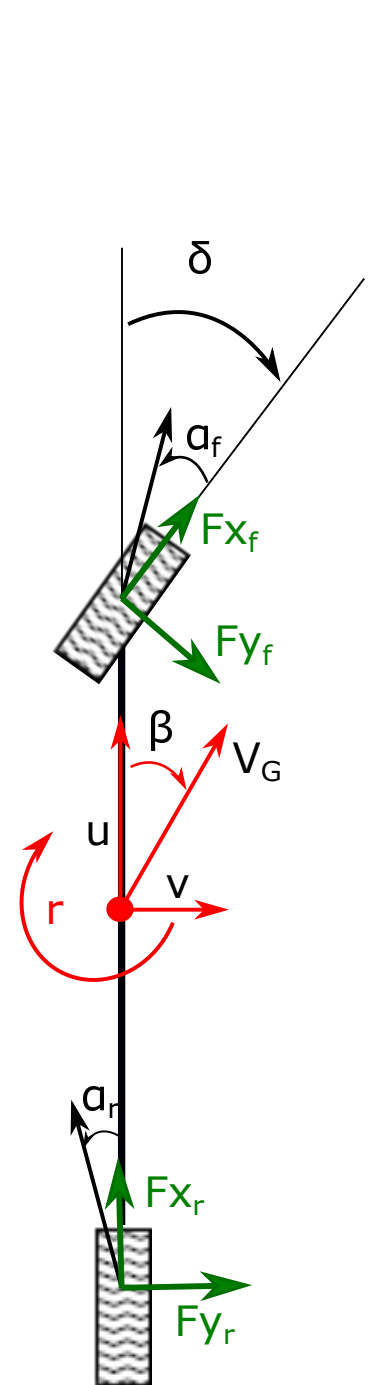

(a) Single-track model

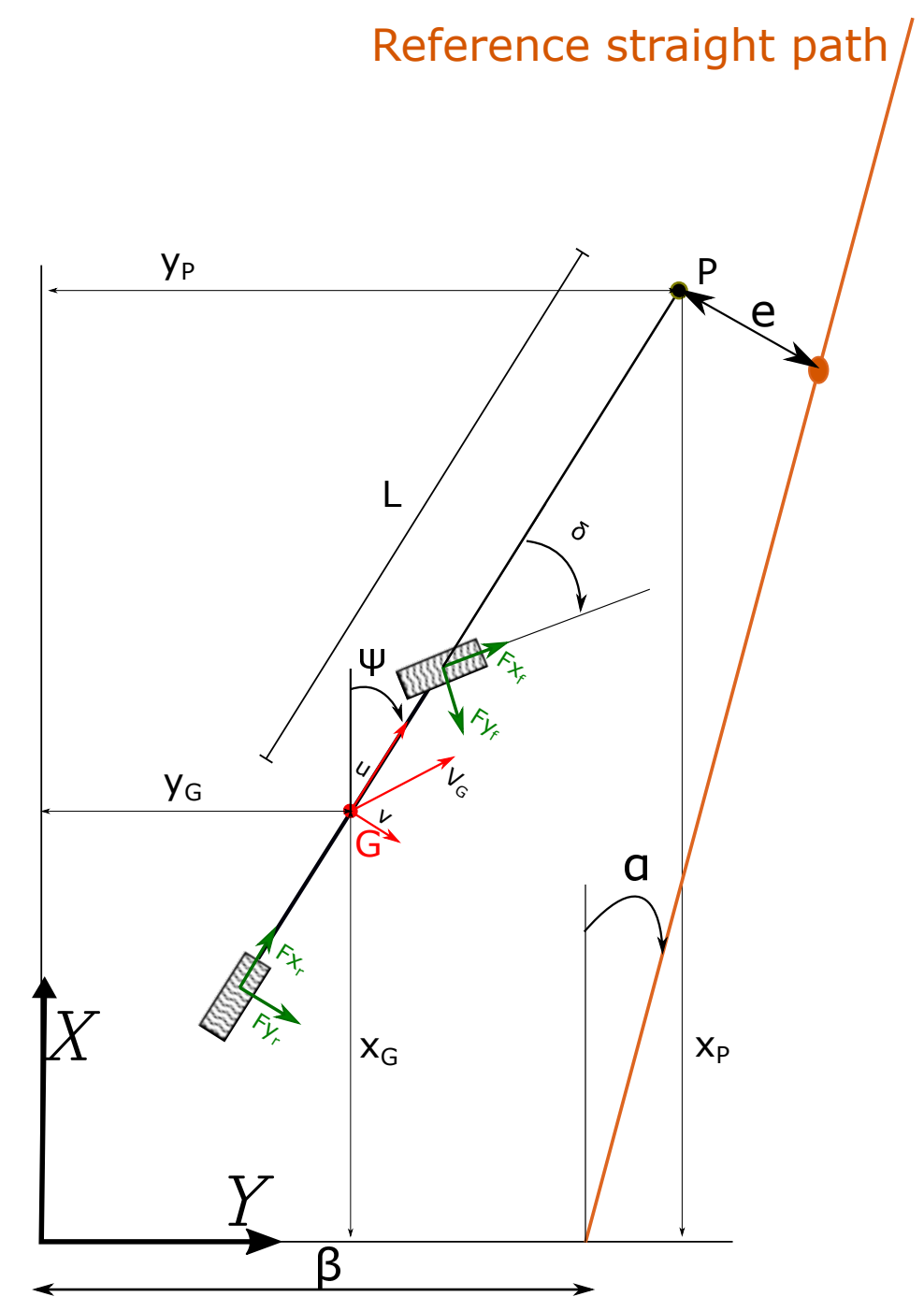

(b) Driver model

Figure 1: Simple car model and simple driver model.

\begin{tabular}{|l|l|l|l|l|}
\hline & B & C & E & D \\
\hline Front & 6.92 & 2.34 & 0.83 & 9493.94 \\
\hline Rear & 10.31 & 2.30 & 1.02 & 9805.56 \\
\hline
\end{tabular}

Table 1: Magic formula tyre coefficients of understeering vehicle.

\begin{tabular}{|l|l|l|l|l|}
\hline & B & C & E & D \\
\hline Front & 10.31 & 2.30 & 1.02 & 10872.50 \\
\hline Rear & 6.92 & 2.34 & 0.83 & 8562.27 \\
\hline
\end{tabular}

Table 2: Magic formula tyre coefficients of oversteering vehicle. 


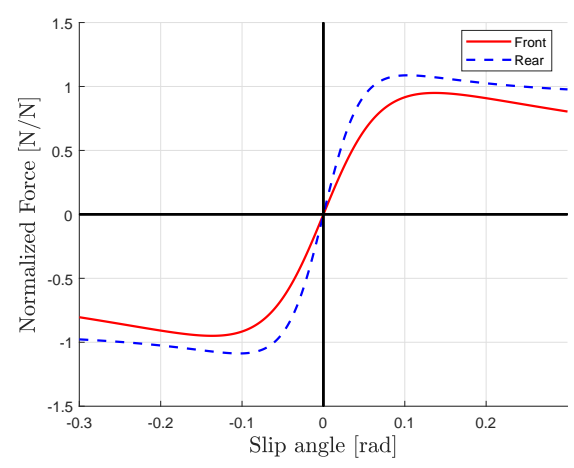

(a) Normalized axle side forces $F_{x 1}, F_{x 2}$

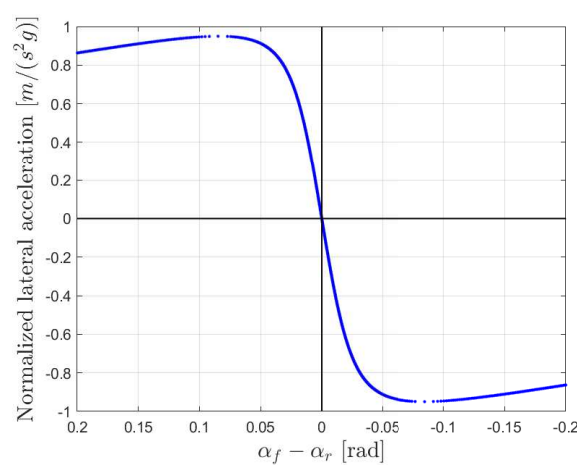

(b) Handling diagram

Figure 2: Handling properties of understeering vehicle.

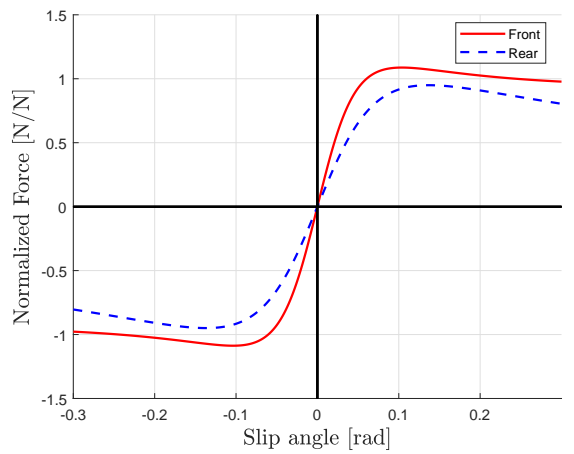

(a) Normalized axle side forces $F_{x 1}, F_{x 2}$

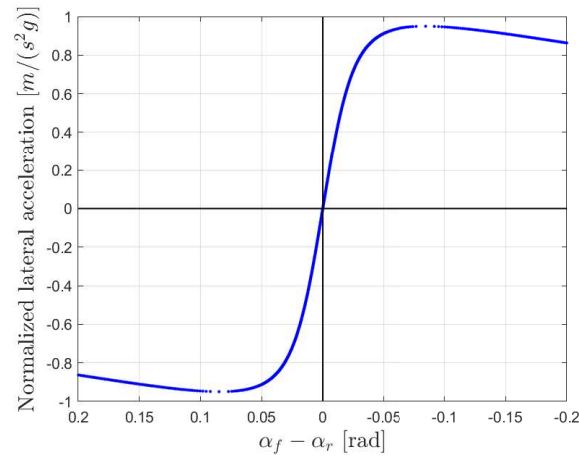

(b) Handling diagram

Figure 3: Handling properties of oversteering vehicle. 
The coefficients that define such configurations are listed in Table 1 and 2 , respectively. The axle characteristics and the corresponding handling diagrams are reported in Fig. 2 and 3. The equations of motion of the single-track model can be derived using the D'Alembert principle and the following equations are derived

$$
\left\{\begin{aligned}
\dot{v} & =\frac{1}{m}\left(F_{y_{f}}+F_{y_{r}}\right)-u r \\
\dot{r} & =\frac{1}{J}\left(a F_{y_{f}}-b F_{y_{r}}\right)
\end{aligned}\right.
$$

\subsection{Simple driver model}

For what concerns the simple driver model, the well known model developed by Weir [37] is used. The driver control logic is illustrated in Fig. 1(b), where a general straight path is illustrated. To simplify the computations, the straight path chosen in this work is the one congruent to the $\mathrm{X}$ axis of the global reference system, so basically the driver has to nullify the lateral position coordinate in the global reference system. The steering action is proportional to the path error computed at a certain distance in front of the vehicle. This distance $L$ is proportional to the longitudinal speed $u$ by setting a fixed preview time $T_{\text {prev }}$, so $L=T_{\text {prev }} u$. The coordinates of the preview point in the global reference system and its speed components can be computed starting from the coordinates of the centre of gravity in the global reference system as follows

$$
\begin{gathered}
\mathbf{P}=\left(x_{P}\right) \mathbf{i}_{0}+\left(y_{P}\right) \mathbf{j}_{0}=\left(x_{G}+L \cos \psi\right) \mathbf{i}_{0}+\left(y_{G}+L \sin \psi\right) \mathbf{j}_{0} \\
\mathbf{V}_{P}=\left(\dot{x}_{P}\right) \mathbf{i}_{0}+\left(\dot{y}_{P}\right) \mathbf{j}_{0}=\left(\dot{x}_{G}-\dot{\psi} L \sin \psi\right) \mathbf{i}_{0}+\left(\dot{y}_{G}+\dot{\psi} L \cos \psi\right) \mathbf{j}_{0}
\end{gathered}
$$

The path error and its time derivative, considering as reference path the $\mathrm{X}$ axis of the global reference system, have the following expressions

$$
\begin{aligned}
& \mathbf{e}=\left(y_{\text {ref }}-y_{P}\right)=-y_{P} \\
& \dot{\mathbf{e}}=\left(\dot{y}_{\text {ref }}-\dot{y}_{P}\right)=-\dot{y}_{P}
\end{aligned}
$$

The steering action of the driver is modelled as a first order system with a time constant $\tau$

$$
\dot{\delta}=\frac{1}{\tau}\left(-\delta+k e+k_{d} \dot{e}\right)
$$

Coupling the vehicle model with the driver model, the final state-space representation of the car \& driver system can be obtained

$$
\left\{\begin{aligned}
\dot{v} & =\frac{1}{m}\left(F_{y_{f}}+F_{y_{r}}\right)-u r \\
\dot{r} & =\frac{1}{J}\left(a F_{y_{f}}-b F_{y_{r}}\right) \\
\dot{\delta} & =\frac{1}{\tau}\left(-\delta+k e+k_{d} \dot{e}\right) \\
\dot{y}_{G} & =u \sin (\psi)+v \cos (\psi) \\
\dot{\psi} & =r
\end{aligned}\right.
$$

In Table 3 the vehicle and driver parameters that have been used in this work are listed.

\subsection{Complex car model}

To check the results obtained with the simple car/simple driver model combination, the complex car/complex driver model combination has been used. The description of the complex car model is provided in the manual of the software Vi-Grade [2]. Basically, the car model is a 14 degrees of freedom multi-body model, in which the bodies are the vehicle chassis and the four wheels. The 


\begin{tabular}{|l|l|}
\hline Mass $m[\mathrm{~kg}]$ & 1938 \\
\hline Moment of inertia $J\left[\mathrm{kgm}^{2}\right]$ & 3992 \\
\hline Front axle distance from center of gravity $a[\mathrm{~m}]$ & 1.628 \\
\hline Rear axle distance from center of gravity $b[\mathrm{~m}]$ & 1.636 \\
\hline Delay time $\tau[\mathrm{s}]$ & 0.2 \\
\hline Preview time $T_{\text {prev }}[\mathrm{s}]$ & 0.5 \\
\hline Proportional gain $k[\mathrm{rad} / \mathrm{m}]$ & 0.02 \\
\hline Derivative gain $k_{d}[\mathrm{rad} /(\mathrm{m} / \mathrm{s})]$ & 0 \\
\hline
\end{tabular}

Table 3: Simple car/simple driver model data.

car body has six degrees of freedom and each wheel has two degrees of freedom, one corresponding to the rolling rotation and one corresponding to the relative displacement between the wheel and the car body. Two reference systems are used, one global, fixed with respect to the ground and one local, fixed with the vehicle, with the origin fixed at the mid point of the front axle, the longitudinal axis is parallel to the vehicle centreline, the normal axis is orthogonal to the ground and directed upwards. The lateral axis is a right-hand reference system with the other mentioned axes. The reference systems are represented in Fig. 4. The directions of both the lateral and vertical axes of the simple car/simple driver model combination are reversed with respect to the corresponding axes of the complex car/complex driver model combination in Fig. 1. In the sequel, the results will be reported using the reference systems of the simple car/simple driver model combination. In Table 4 the main parameters of the complex car model are listed. Camber angles are positive if the wheels are inclined towards the chassis. The steering ratio and

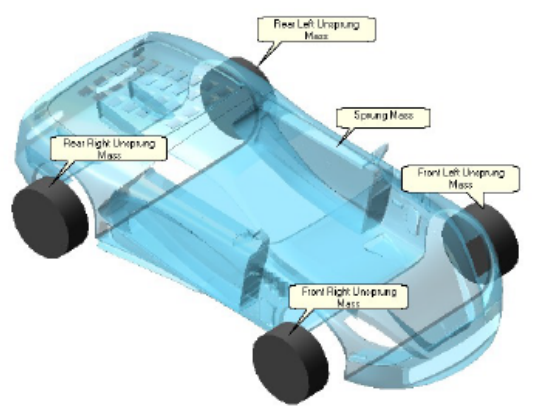

(a) 14 dof vehicle model

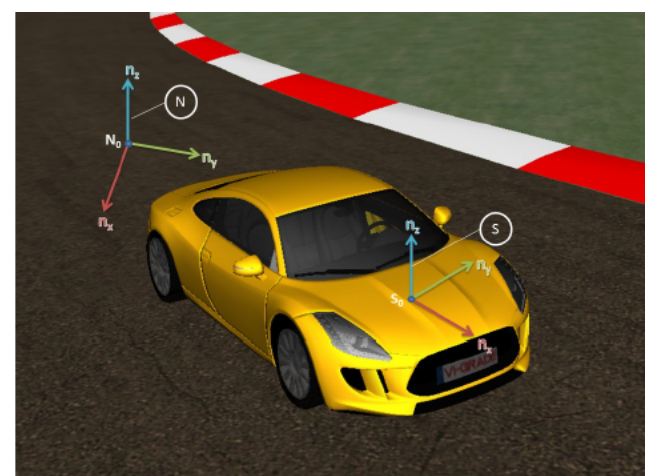

(b) Reference systems

Figure 4: Vi-Grade vehicle modelling framework.

the wheel angles are referred to the equilibrium position of the car running straight at constant speed. Due to the rolling and pitching motions these quantities can vary, the software takes into account such phenomenon. The vehicle that is modelled is a rear wheel drive $\mathrm{E}$ segment passenger car equipped with a limited slip differential. In order to make the complex car model more similar to the simple car model, the differential is changed to a simple open differential. The modelling of the tyre is done by using the complete Pacejka Magic Formula, whose coefficients correspond to the parameters in Table 1 and 2. The aerodynamic forces have been reduced, in order to have the possibility to reach very high longitudinal velocities, actually at such speeds important nonlinear behaviour, e.g. bifurcations, occur. 


\begin{tabular}{|l|l|}
\hline Chassis mass $\left(m_{c}\right)$ & $1691 \mathrm{~kg}$ \\
\hline Chassis inertia moment $\left(J_{c}\right)$ & $3340 \mathrm{kgm}$ \\
\hline Front tire-hub mass $\left(m_{f}\right)$ & $52 \mathrm{~kg}$ \\
\hline Front tire-hub inertia moment $\left(J_{f}\right)$ & $1.35 \mathrm{kgm}^{2}$ \\
\hline Rear tire-hub mass $\left(m_{r}\right)$ & $40 \mathrm{~kg}$ \\
\hline Rear tire-hub inertia moment $\left(J_{r}\right)$ & $1.04 \mathrm{kgm}^{2}$ \\
\hline Fuel mass $\left(m_{\text {fuel }}\right)$ & $63 \mathrm{~kg}$ \\
\hline Fuel mass longitudinal position $\left(x_{\text {fuel }}\right)$ & $-2.8 \mathrm{~m}$ \\
\hline Center of gravity distance from the front axle $(a)$ & $1.628 \mathrm{~m}$ \\
\hline Center of gravity distance from the front axle $(b)$ & $1.636 \mathrm{~m}$ \\
\hline Wheelbase $(l)$ & $2.97 \mathrm{~m}$ \\
\hline Front track $\left(t_{f}\right)$ & $2.97 \mathrm{~m}$ \\
\hline Rear track $\left(t_{r}\right)$ & $2.97 \mathrm{~m}$ \\
\hline Steering ratio & 14.54 \\
\hline Toe angle Front Left (static) & $-0.36 \mathrm{deg}$ \\
\hline Toe angle Front Right (static) & $0.36 \mathrm{deg}$ \\
\hline Toe angle Rear Left (static) & $0.165 \mathrm{deg}$ \\
\hline Toe angle Rear Right (static) & $-0.165 \mathrm{deg}$ \\
\hline Camber angle Front Left (static) & $-0.35 \mathrm{deg}$ \\
\hline Camber angle Front Right (static) & $-0.35 \mathrm{deg}$ \\
\hline Camber angle Rear Left (static) & $-0.9 \mathrm{deg}$ \\
\hline Camber angle Rear Right (static) & $-0.9 \mathrm{deg}$ \\
\hline
\end{tabular}

Table 4: Vi-Grade vehicle model: data.

\subsection{Complex driver model}

The Virtual Driver provided by the Vi-Grade software has been used as it seems more accurate with respect to the single-point preview driver model (simple driver model). The Virtual Driver logic is based on an MPC control logic. MPC stands for Model Predictive Control and it is a control algorithm that optimizes a control variable in order to minimize a cost function over a finite-time horizon. In order to have a better explanation of the driver control logic the reader is invited to consult the Vi-Grade manual [2].

\subsection{Perturbation method}

When simulating a dynamical system, in order to evaluate the stability of an equilibrium position or the stability of a limit cycle, a perturbation has to be generated. In this paper two strategies have been used to perturb the car \& driver dynamical system. The first one is simply an initial lateral displacement. It has been used to evaluate the stability of straight running. The second strategy applies a lateral impulse force to the vehicle. The second strategy has been introduced since the first one does not work properly for the complex or human driver model. Actually, during tests at the driving simulator, we found that recovering a sudden lateral displacement applied to the vehicle resulted in a simple task for the driver. In fact, no input energy was associated to such a disturbance that was just a re-positioning of the vehicle in a displaced initial position on the road. The driver did not feel any lateral acceleration and this was perceived as an unrealistic situation. In other words, the initial condition at the driving simulator, as it is comes from the first strategy, was not associated to an actual disturbance by the driver. So we decided to adopt the second strategy. The perturbation scheme and impulse force shape are reported in Fig. 5. The magnitude of the force has been fixed and equal to $F_{\text {dist }}=-10000 \mathrm{~N}$, congruent 
with the global reference system defined in the previous section. The parameter chosen to vary the magnitude of the perturbation has been the arm of the force with respect to the vehicle centre of gravity. Increasing the arm of the external force results in an increasing moment acting on the system, thus giving bigger perturbation.
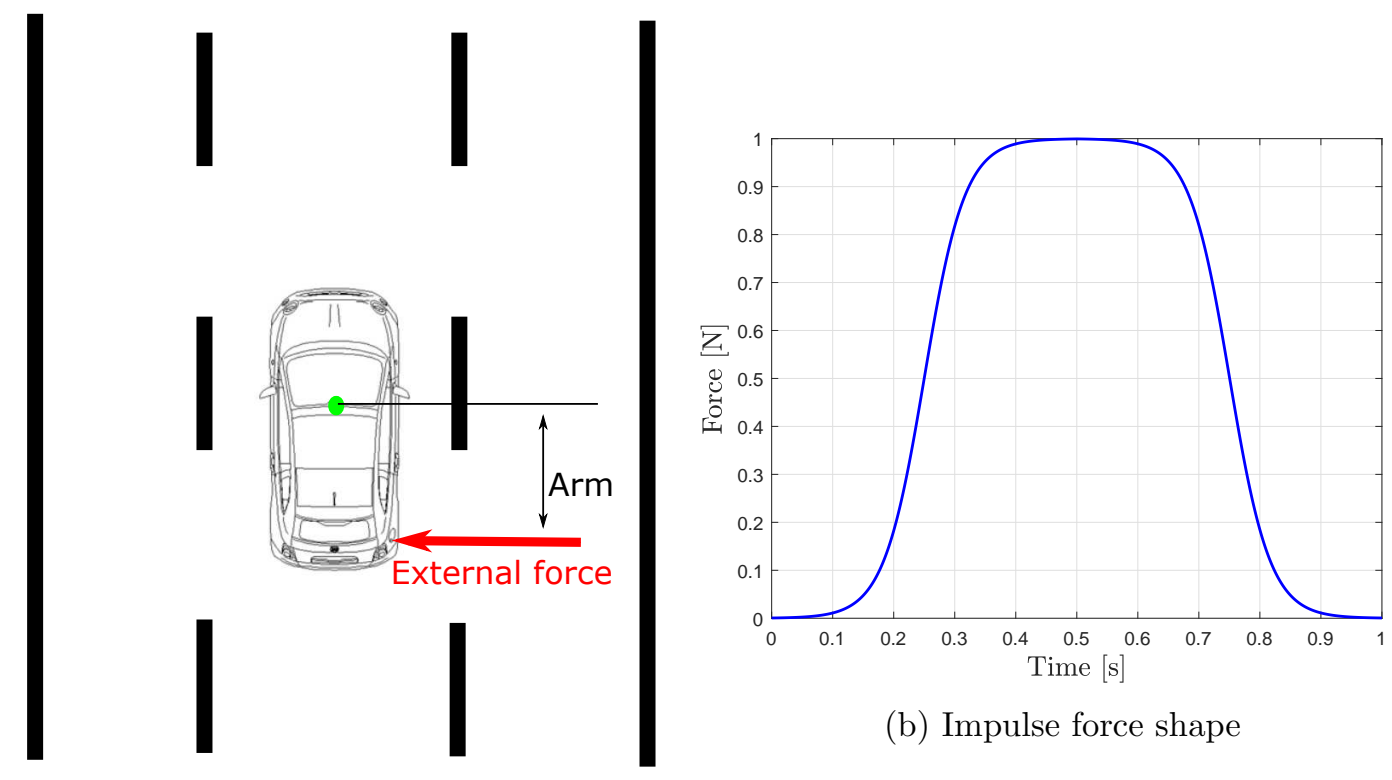

(b) Impulse force shape

(a) Impulse force perturbation

Figure 5

\section{Bifurcation analysis - combination of simple car model and simple driver model}

In this section we take into account the simple car/simple driver model combination. To evaluate the related dynamical behaviour, bifurcation theory has been used $[16,32,11]$. We perform our analysis for different values of the longitudinal speed, that thus becomes our bifurcation parameter. Bifurcation diagrams have been produced using Matcont [9]: in Fig. 6a, 7a we report a three-dimensional projection of the bifurcation diagram of our 5 dimensional simple system. Solid blue lines indicate stable equilibria or stable limit cycles, dashed red lines indicate unstable equilibria or unstable limit cycles. A critical speed, i.e. a bifurcation speed, is found in either cases. At longitudinal speeds lower than the bifurcation one, an unstable limit cycle exists in the state-space. The unstable limit cycle repulses the state space trajectories. The motion of the vehicle can be repulsed towards the straight running equilibrium or can diverge, depending on the initial state-space condition. The amplitude of the unstable limit cycle increases as the speed decreases, so at lower speeds the perturbation able to make unstable the response of the vehicle has to be very high, as common sense suggests.

In Fig. $6 \mathrm{~b}$ and Fig. $7 \mathrm{~b}$ projections of the bifurcation diagrams in the lateral positionlongitudinal speed plane are reported. To visualize better the trend of the limit cycles, the maximum value of the lateral position variable reached during the cycle is reported. In both cases, namely understeering and oversteering, the simple car/simple driver model predicts a stability loss of the desired trajectory, due to a subcritical Hopf bifurcation. In the oversteering case, the stability loss occurs at a longitudinal speed of nearly $20 \mathrm{~m} / \mathrm{s}$. In the understeering case, the stability loss occurs at a longitudinal speed of nearly $100 \mathrm{~m} / \mathrm{s}$. Passed those values, the 
equilibria are unstable and the driver is not able to find another stable motion, so such types of bifurcations are catastrophic.

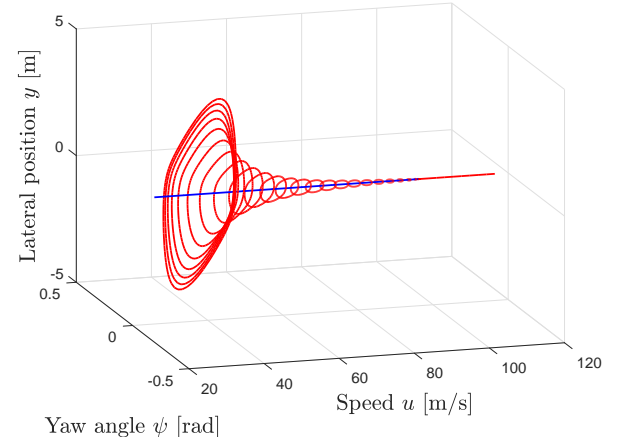

(a) State-space bifurcation diagram

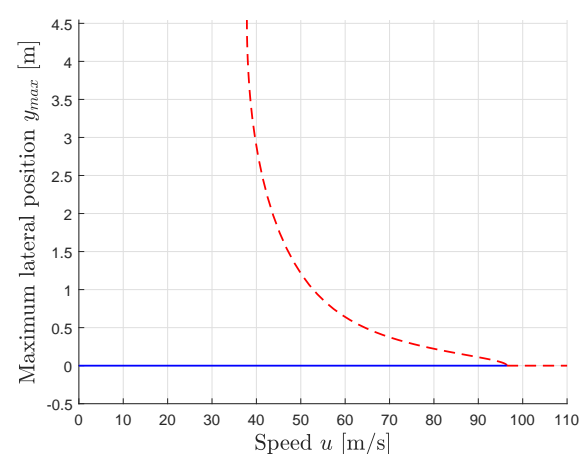

(b) Maximum value of the lateral position

Figure 6: Combination of simple car model and simple driver model - understeering configuration: Bifurcation analysis varying longitudinal speed. Data in Tab.1 and Tab.3.

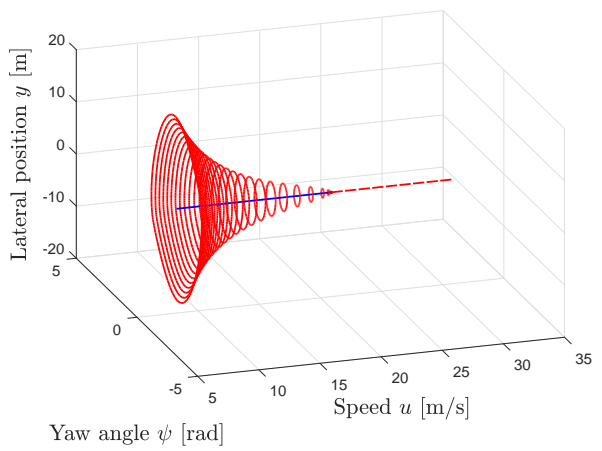

(a) State-space bifurcation diagram

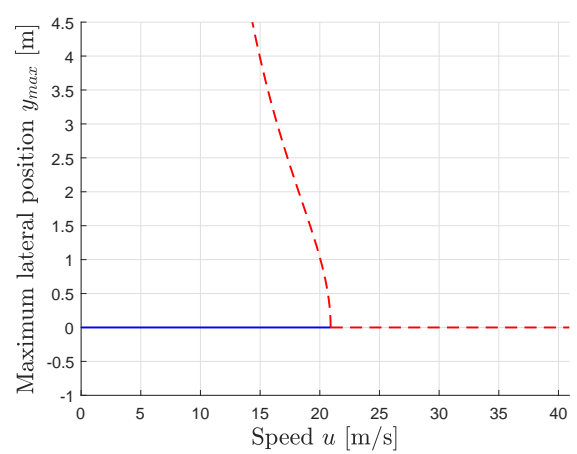

(b) Maximum value of the lateral position

Figure 7: Combination of simple car model and simple driver model - oversteering configuration: Bifurcation analysis varying longitudinal speed. Data in Tab.2 and Tab.3.

We check now the existence of a critical, or bifurcation, speed by resorting to time history simulations. In Fig. 8 the dynamical response of the simple car/simple driver model combination in understeering configuration is shown. Due to a small perturbation, the dynamical behaviour of the system changes its nature as the longitudinal speed is increased. The starting lateral position is equal to $y_{0}=0.1 \mathrm{~m}$. Increasing the speed, the response is less and less damped, but stable, till a certain critical speed, if such a speed is passed, the response is unstable. The system has two low frequency eigenmodes at $0.35 \mathrm{~Hz}$ and $1 \mathrm{~Hz}$; increasing the speed, the magnitude of the component at $1 \mathrm{~Hz}$ increases because this is the eigenmode that causes instability.

The simulations mentioned above have been done providing to the system a perturbation of low magnitude. It is important to investigate how the behaviour of the system changes by increasing the magnitude of the perturbation. The perturbation strategy is now the second one described in the previous section, an impulse force is applied at a certain distance from the centre of gravity. Let us consider Fig. 9. We want to see whether the stability depends on the amplitude of the disturbance. The analysis has been done at $70 \mathrm{~m} / \mathrm{s}$, a velocity for which the response to a small perturbation is stable. If the perturbation is too high, the response is unstable. The driver is able to restore the straight running condition till a certain threshold, which is found in Fig. 


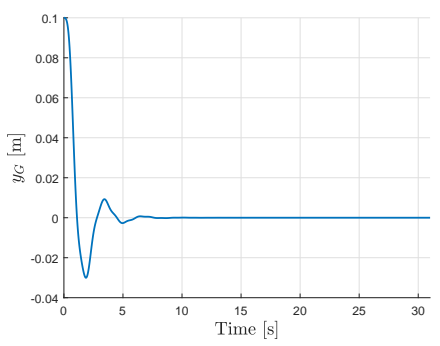

(a) $u=80 \mathrm{~m} / \mathrm{s}, \mathrm{c} \cdot \mathrm{g}$. position

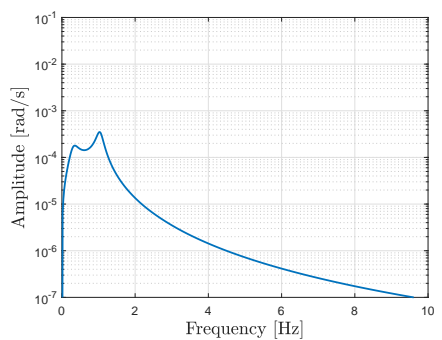

(d) $u=80 \mathrm{~m} / \mathrm{s}$, Yaw Rate FFT

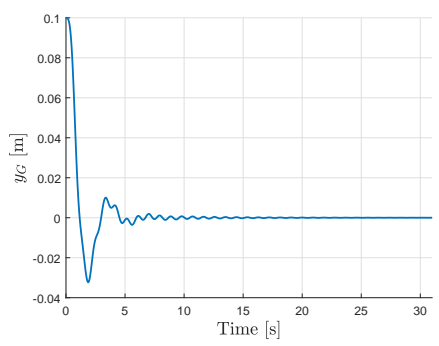

(b) $u=90 \mathrm{~m} / \mathrm{s}$, c.g. position

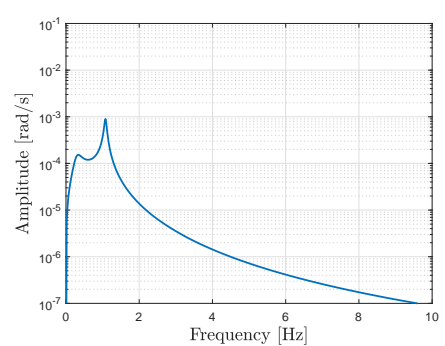

(e) $u=90 \mathrm{~m} / \mathrm{s}$, Yaw Rate FFT

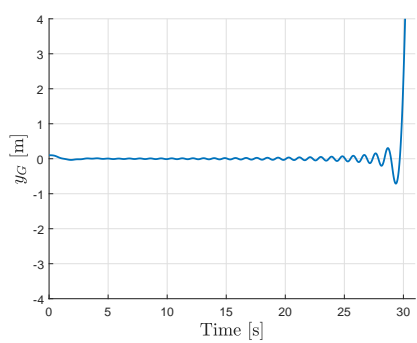

(c) $u=100 \mathrm{~m} / \mathrm{s}$, c.g. position

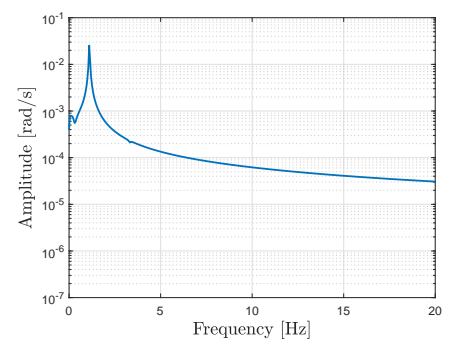

(f) $u=100 \mathrm{~m} / \mathrm{s}$, Yaw Rate FFT

Figure 8: Combination of simple car model and simple driver model - understeering configuration: Estimation of the bifurcation longitudinal speed by applying a small initial condition (first perturbation strategy). Data in Tab.1 and Tab.3.

9. We do not show the threshold for other speeds here because no additional information could be provided. The impulse force starts after $1 \mathrm{~s}$ and lasts $1 \mathrm{~s}$.

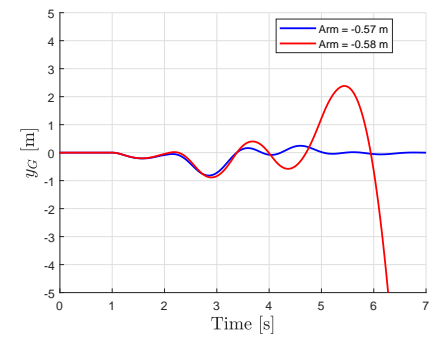

(a) c.g. position

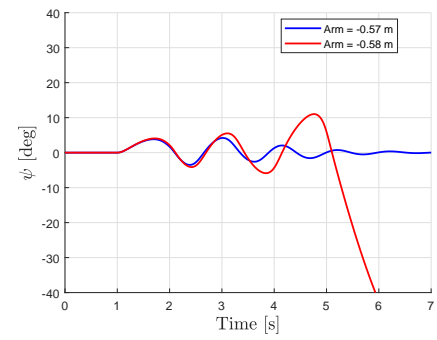

(b) Yaw angle

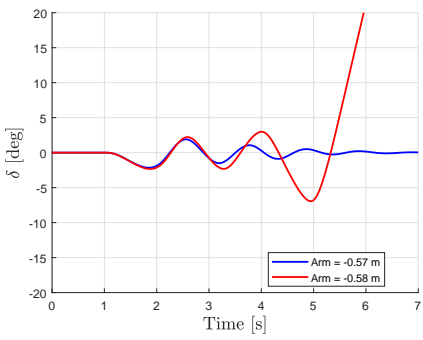

(c) Steering angle

Figure 9: Combination of simple car model and simple driver model - understeering configuration. A slight increase of disturbance (second perturbation strategy) causes instability, $u=70 \mathrm{~m} / \mathrm{s}$. Data in Tab.1 and Tab.3.

The simulations presented in Fig. 8 and in Fig. 9 have been done considering the understeering configuration. The corresponding investigations for oversteering configuration are reported in Fig.10 and in Fig.11. The qualitative behaviour is equal to the one observed with understeering configuration, but the main difference is the speed range at which the system loses stability, which is lower with respect to the one of understeering configuration. Similar theoretical results concerning the subcritical Hopf bifurcation as a source of instability for the car \& driver system have been obtained in other papers $[6,5,7,19]$ and [20]. Qualitatively the results are here confirmed.

In Fig. 12 different bifurcations are obtained by varying the driver parameters. In Fig. 12a it can be noted that increasing the lag or delay time of the driver, the bifurcation speed decreases. 


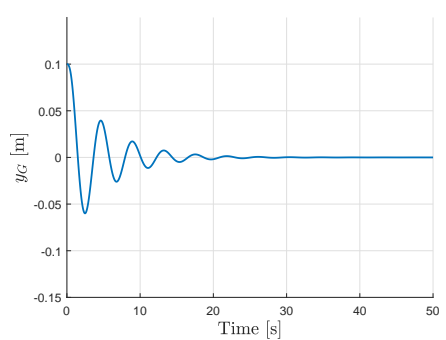

(a) $u=15 \mathrm{~m} / \mathrm{s}$, c.g. position

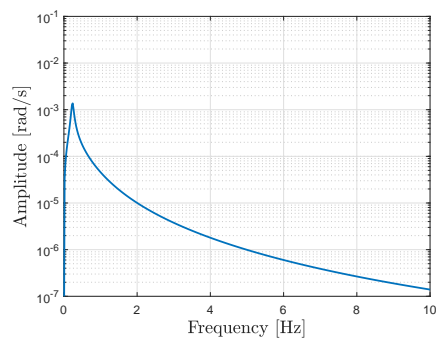

(d) $u=15 \mathrm{~m} / \mathrm{s}$, Yaw Rate FFT

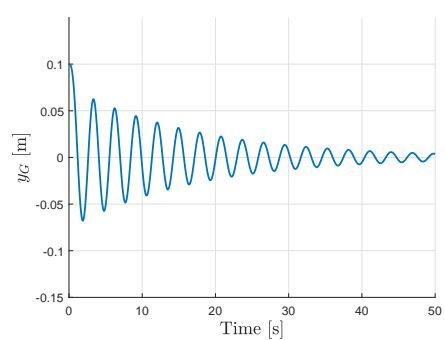

(b) $u=20 \mathrm{~m} / \mathrm{s}, \mathrm{c} . \mathrm{g}$. position

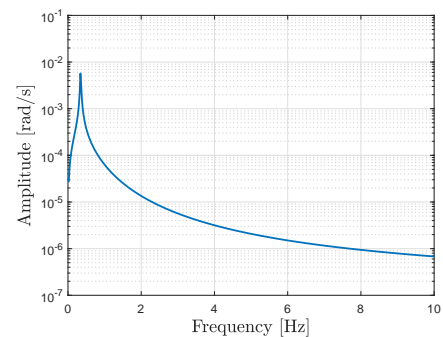

(e) $u=20 \mathrm{~m} / \mathrm{s}$, Yaw Rate FFT

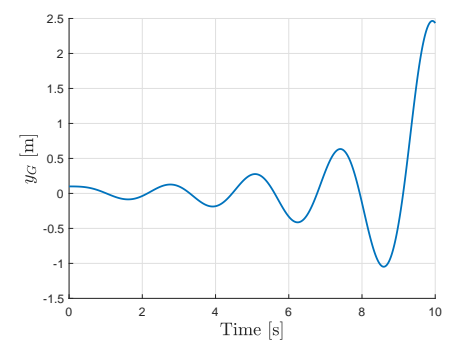

(c) $u=25 \mathrm{~m} / \mathrm{s}$, c.g. position

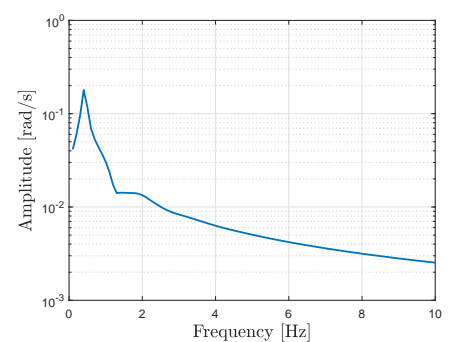

(f) $u=25 \mathrm{~m} / \mathrm{s}$, Yaw Rate FFT

Figure 10: Combination of simple car model and simple driver model - oversteering configuration: Estimation of the bifurcation longitudinal speed by applying a small initial condition (first perturbation strategy). Data in Tab.2 and Tab.3.

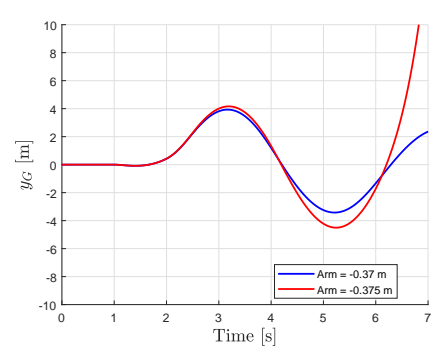

(a) c.g. position

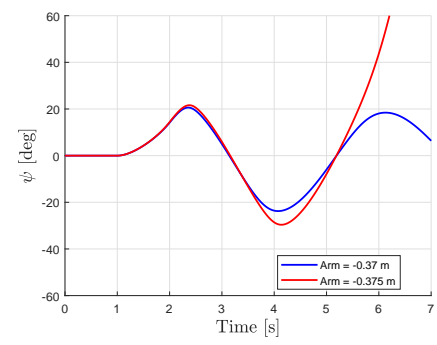

(b) Yaw angle

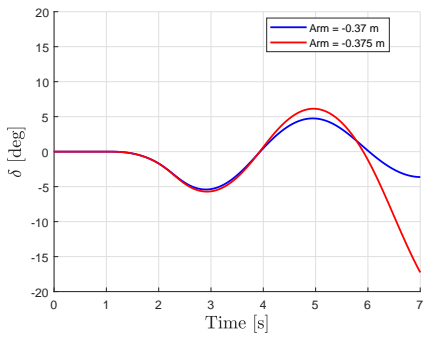

(c) Steering angle

Figure 11: Combination of simple car model and simple driver model - oversteering configuration. A slight increase of disturbance (second perturbation strategy) causes instability, $u=15 \mathrm{~m} / \mathrm{s}$. Data in Tab.2 and Tab.3. 
In Fig. 12b the Lyapunov coefficient is given as function of the proportional gain of the driver. The Lyapunov coefficient describes the type of Hopf bifurcation. If the coefficient is positive, the Hopf bifurcation is subcritical, if it is negative, the Hopf bifurcation is supercritical. It can be seen that simply varying the proportional gain, the Hopf bifurcation changes its nature from supercritical to subcritical. This is consistent with and substantiates what was found in [6], i.e. either subcritical or supercritical bifurcation may occur, given a car, depending on the driver parameters.

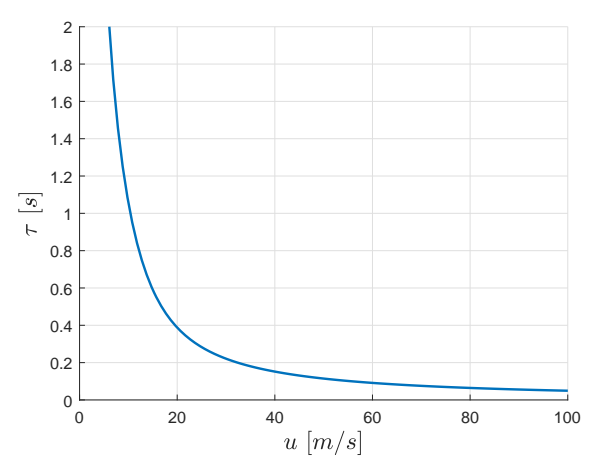

(a) Bifurcation speed as function of the delay time of the driver

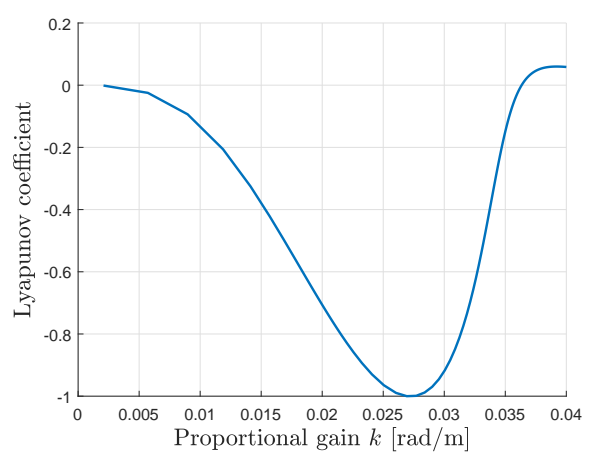

(b) Hopf type bifurcation depending on Lyapunof coeff. as function of driver control gain

Figure 12: Combination of simple car model and simple driver model. Influence of driver parameters. Data in Tab.1 and Tab.3.

Since many different bifurcations may occur, we wanted to investigate whether with more complex vehicle systems, bifurcations phenomena do exist.

\section{Bifurcation analysis - combination of complex car model and complex driver model}

The same simulations performed with the simple car/simple driver model combination were performed with the complex car/complex driver model combination. We investigated the presence of Hopf bifurcations by varying the longitudinal speed. Additionally, the presence of stable or unstable limit cycles was studied. This procedure has been done both considering an understeering car or an oversteering one.

Referring to the understeering configuration, Fig. 13 shows the behaviour of the car \& driver system as speed increases. For longitudinal speeds lower than $75 \mathrm{~m} / \mathrm{s}$, the system is asymptotically stable towards the straight running condition. Increasing the speed, the lateral motion of the car centre of gravity is less damped. The time response has a frequency content with only one main component at $0.2 \mathrm{~Hz}$. For longitudinal speeds equal or higher than $78 \mathrm{~m} / \mathrm{s}$, the system is not asymptotically stable, actually it continues oscillating with a main component of the frequency spectrum at $0.18 \mathrm{~Hz}$. The motion is not periodic, but it looks chaotic. By inspection of the spectrum of the yaw rate at a longitudinal speed equal to $100 \mathrm{~m} / \mathrm{s}$ it can be noted that the main component of the spectrum has a frequency that is decreased to $0.14 \mathrm{~Hz}$ and other components with significant amplitudes are present at higher frequencies. The irregularity of the time series and the shape of the frequency spectrum indicates that the motion is fully chaotic. The amplitude of this chaotic motion is not very high, having a range of $\pm 0.15 \mathrm{~m}$ of lateral displacement with respect to the reference straight path. Nevertheless if this motion would be replicated in a real environment, it would be sensed by the human driver and the driving experi- 


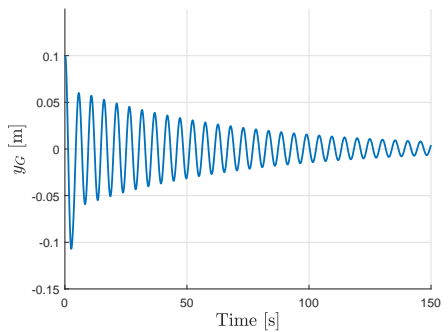

(a) $u=75 \mathrm{~m} / \mathrm{s}$, c.g. position

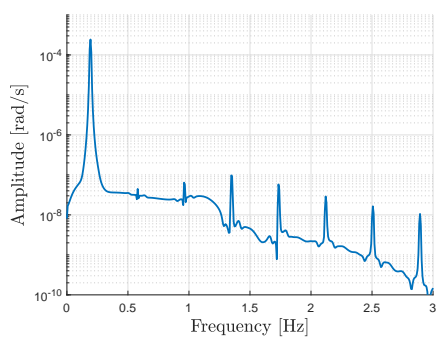

(d) $u=75 \mathrm{~m} / \mathrm{s}$, Yaw Rate FFT

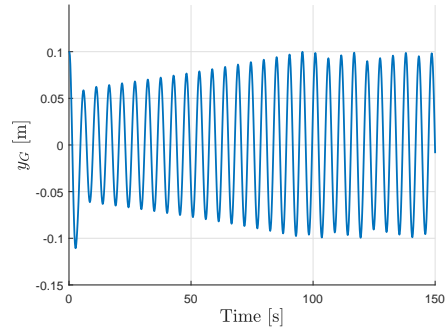

(b) $u=78 \mathrm{~m} / \mathrm{s}$, c.g. position

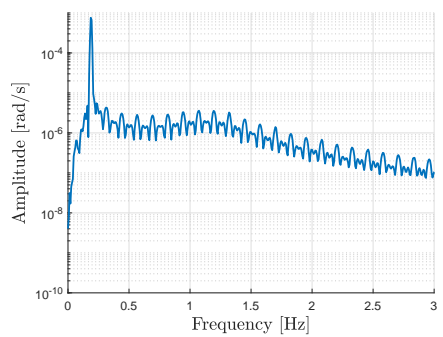

(e) $u=78 \mathrm{~m} / \mathrm{s}$, Yaw Rate FFT

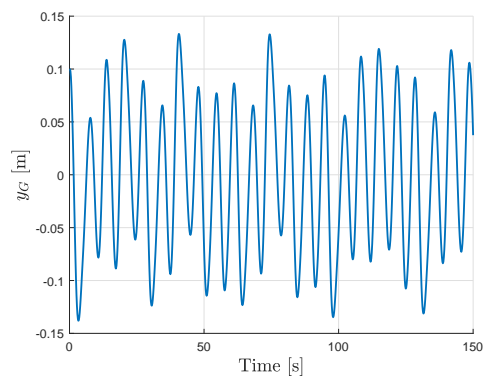

(c) $u=100 \mathrm{~m} / \mathrm{s}$, c.g. position

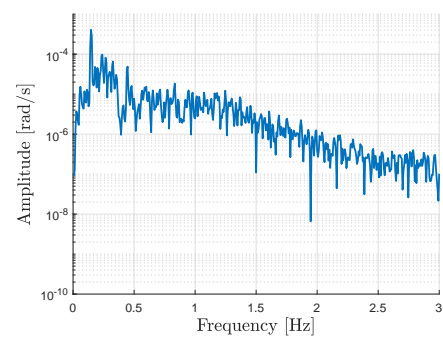

(f) $u=100 \mathrm{~m} / \mathrm{s}$, Yaw Rate

Figure 13: Combination of complex car model and complex driver model - understeering configuration: Estimation of the bifurcation longitudinal speed by applying a the second perturbation strategy. Data in Tab.4. and in [2]

ence would be negatively influenced. In [20] a chaotic motion was found after a Hopf bifurcation for a simple vehicle model, this occurrence is here confirmed for a complex car model.

After having estimated the bifurcation speed, the response due to different magnitudes of perturbation is analyzed. Simulations have been performed at $70 \mathrm{~m} / \mathrm{s}$ and $90 \mathrm{~m} / \mathrm{s}$, such values of longitudinal velocity occur before and after the bifurcation speed respectively. The results are reported in Fig. 14 and Fig. 15. Note that the scale of the vertical axis in these figures is much wider that the one of Fig.13. In both of the tests there is a threshold value of the perturbation that makes the type of response changing form stable to unstable (red curve in the figures). On the other hand, in both of the cases small perturbations are absorbed by the system (blue curves), either coming back to an equilibrium (Fig. 14) or to a small-amplitude attractor (Fig. $15)$.

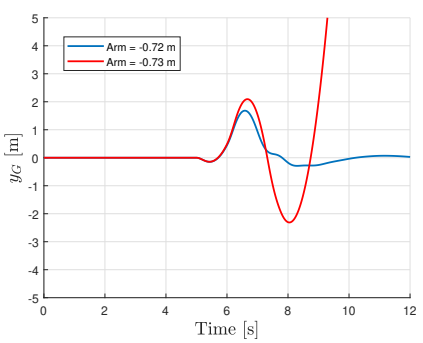

(a) Lateral position

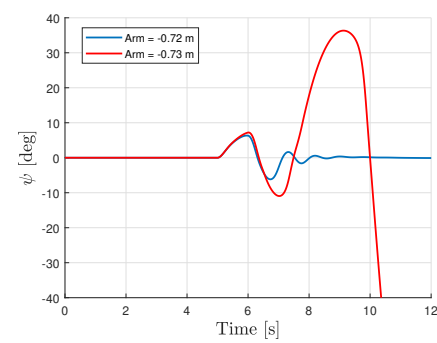

(b) Yaw angle

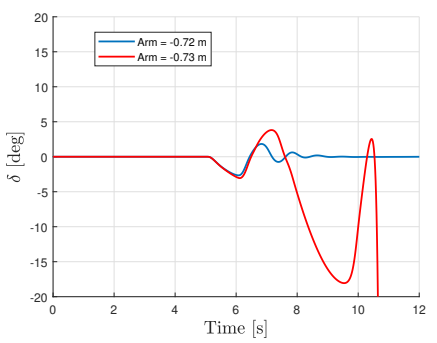

(c) Steering angle

Figure 14: Combination of complex car model and complex driver model - understeering configuration. A slight increase of disturbance (second perturbation strategy) causes instability, $u=70$ $m / s$. Data in Tab.4. and in [2].

A bifurcation diagram like the one in Fig.6b cannot be obtained for the combination of 


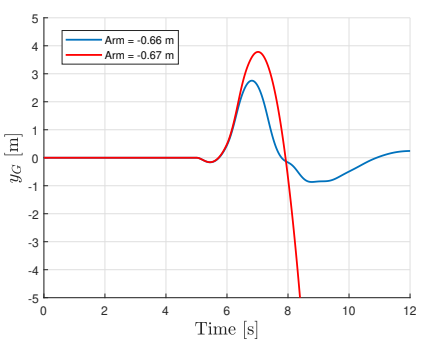

(a) Lateral position

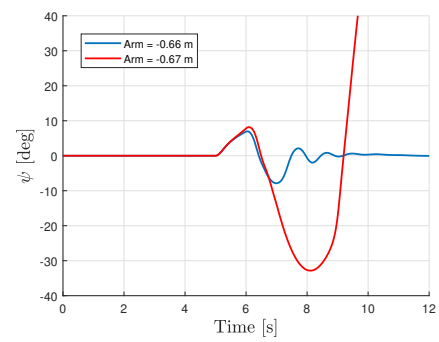

(b) Yaw angle

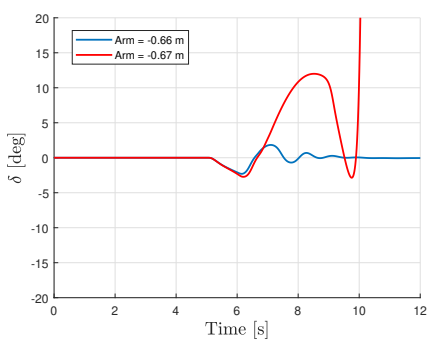

(c) Steering angle

Figure 15: Combination of complex car model and complex driver model. Understeering configuration. A slight increase of disturbance (second perturbation strategy) causes instability, $u=90$ $\mathrm{m} / \mathrm{s}$. Data in Tab.4. and in [2].

complex car model and complex driver model. Actually, the equations describing the complex driver model are not available for commercial reasons. Nevertheless based on the simulations results, the properties of the straight running equilibrium and the existence of stable or unstable limit cycles can be estimated. This estimation is presented in Fig. 16 which corresponds to Fig.6b. The oscillations approaching the bifurcation are less and less damped (Fig. 13, panels $\mathrm{a}$ and $\mathrm{b}$ ), after a small perturbation, the system evolves towards the equilibrium or a nearby attractor (blue curve in Fig.14 and 15, respectively). We identify the type of bifurcation that causes the loss of stability of the desired equilibrium as a supercritical Hopf bifurcation. After this bifurcation, the new born periodic attractor undergoes a Neymark-Sacker bifurcation (in which multiple armonics are created) and the invariant torus is finally destroyed giving rise to the strange attractor identified in Fig. 13(c). We also conjecture the presence of an unstable limit cycle that is sufficiently far -not involved- in the bifurcation. Such limit cycle should be present, causing the response to be unstable if the perturbation is high enough (red curves in in Fig. 14 and 15).

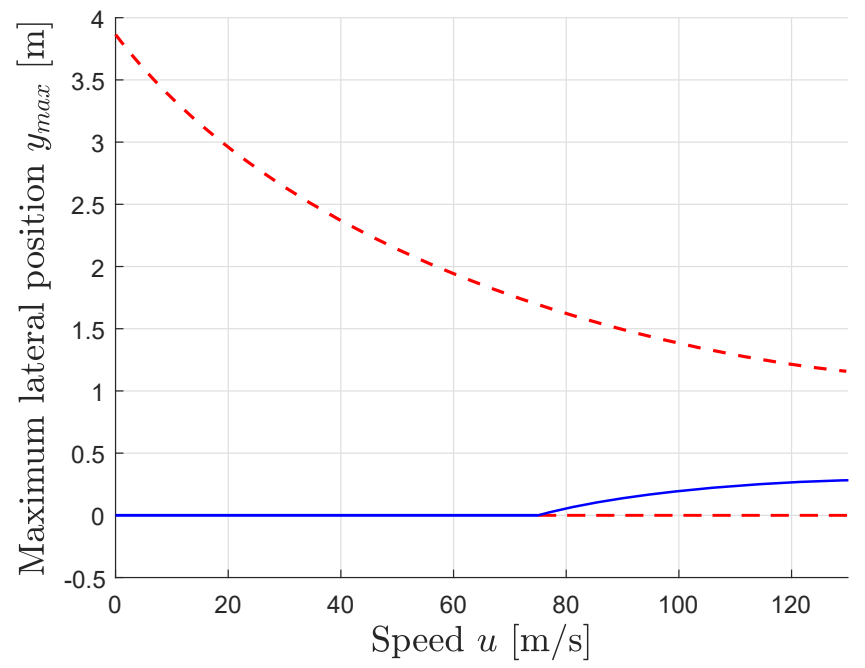

Figure 16: Combination of complex car model and complex driver model. Understeering configuration. Approximation of the maximum value of the lateral position in the bifurcation diagram. Upper dashed line: amplitude of the unstable limit cycle.

The analysis concerning the oversteering configuration qualitatively matches the one obtained with the simple car/simple driver model combination. In Fig. 17 the simulations obtained by 
providing a small perturbation to the system and increasing the longitudinal speed are reported. The bifurcation speed is in between $30 \mathrm{~m} / \mathrm{s}$ and $35 \mathrm{~m} / \mathrm{s}$, due to the change in the stability properties of the straight running equilibrium. After the bifurcation, no stable motions has been appreciated. The bifurcation is thus a catastrophic bifurcation. The presence of oscillations lead us to classify the bifurcation as a subcritical Hopf.

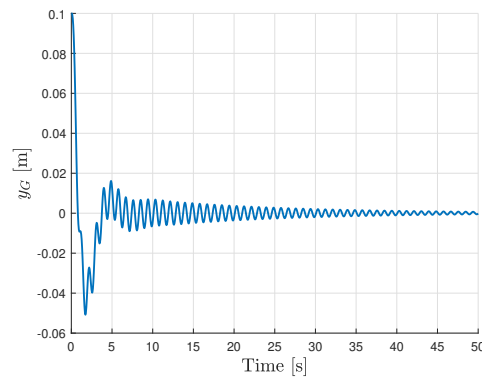

(a) $u=30 \mathrm{~m} / \mathrm{s}$, c.g. position

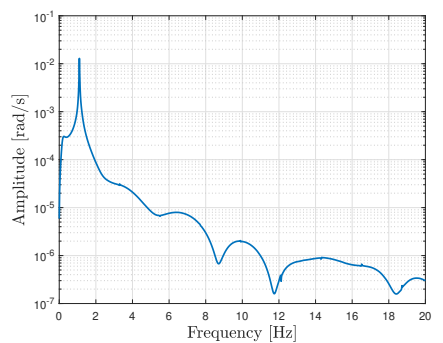

(c) $u=30 \mathrm{~m} / \mathrm{s}$, Yaw Rate FFT

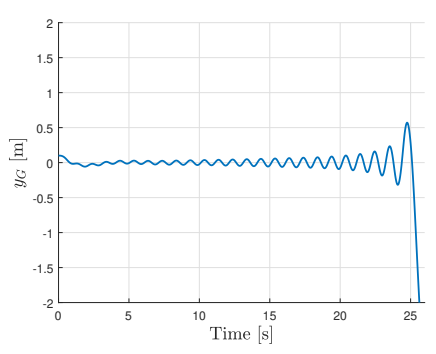

(b) $u=35 \mathrm{~m} / \mathrm{s}$, c.g. position

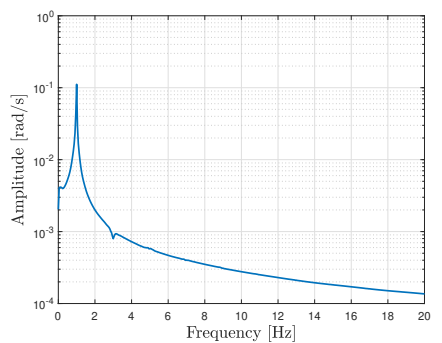

(d) $u=35 \mathrm{~m} / \mathrm{s}$, Yaw Rate FFT

Figure 17: Combination of complex car model and complex driver model. Oversteering configuration. Estimation of the bifurcation longitudinal speed. Data in Tab.4 and in [2]

We have found that stability can be lost during straight running by increasing the speed for the complex car / complex driver model combination. Furthermore the presence of unstable limit cycles has been confirmed. Additionally a chaotic motion has been highlighted.

\section{Driving simulator}

To validate the theoretical results, we performed experimental tests using a professional dynamic driving simulator. The driving simulator, shown in Fig. 18, has been provided by Danisi Engineering [1]. The simulator has 9 degrees of freedom and it can rotate and translate to reproduce as accurately as possible the accelerations of the vehicle. The vehicle dynamics fidelity and the driving experience has been validated by professional drivers. This is due to low latence of the simulator, less than $20 \mathrm{~ms}$. Results obtained using the driving simulator can be found in real driving conditions with high probability. The vehicle model implemented in the simulator has 14 degrees of freedom and corresponds to the complex car model we presented previously.

\section{Driving simulator experimental tests}

The tests have been divided into two different sets, the first set considers the understeering vehicle, the second set considers the oversteering vehicle. The loop is closed by an actual human driver. The tests follow the same scheme used in the previous sections. First, the bifurcation speed has been estimated by increasing the longitudinal speed while the system is excited by a 


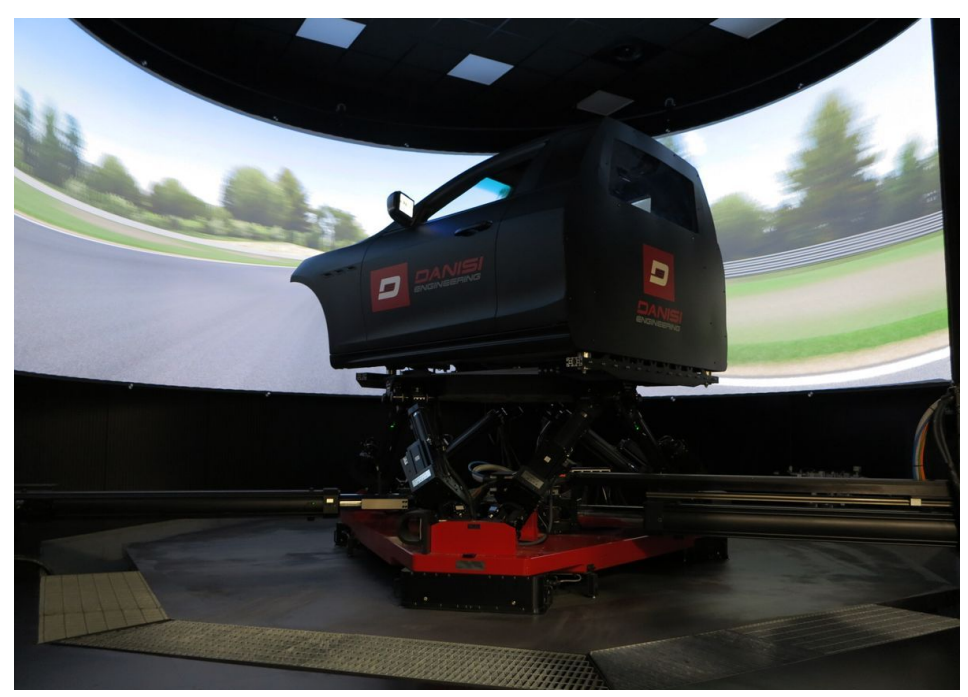

Figure 18: Danisi Engineering dynamic driving simulator.

low magnitude perturbation (that is, the irregularity of the road surface). Secondly, the presence of an unstable limit cycle has been investigated by doing multiple tests at constant speed with perturbations of increasing amplitude.

The first vehicle configuration is understeering. The longitudinal speed has been increased till $120 \mathrm{~m} / \mathrm{s}$, and no unstable behaviour has been observed. The results corresponding to a longitudinal speed of $90 \mathrm{~m} / \mathrm{s}$ are reported in Fig. 19. It can be noted that it is present a low amplitude chaotic motion, as it was observed doing simulations with the complex car/complex driver model combination. Unfortunately, in this case it is not possible to establish a speed range in which the bifurcation occurs, since the perturbation (irregularity of the road surface) is always present. Thus, understanding if the chaotic motion is caused by the road irregularity or by the driver inability to follow a straight path requires a deeper investigation, using a different set-up in the driving simulator. This is left to a forthcoming research which involves an accurate modeling of ply-steer and conicity [23]. Understanding this difference can be important at a practical level but the aim of this paper is to have a broad overview on the existence of bifurcations.

To investigate the existence of an unstable limit cycle, in Fig. 20 and 21 experimental tests obtained by varying the perturbation magnitude at two different constant speeds are reported. Keeping constant the longitudinal speed, and varying the magnitude of perturbation provided to the system, the response of the car \& driver system passes from being stable to being unstable. Considering the state variables trends during the impulse force application (that starts at $1 \mathrm{~s}$ and finishes at $2 \mathrm{~s}$ ), it can be noted that, at the very beginning, the difference between the two responses is not high, but it is sufficient to change the type of the subsequent time history.

This suggests the presence of an unstable limit cycle. Considering the resume of all the experimental tests reported in Fig.22, it can be seen that the boundary between stable and unstable tests follows the trend of the amplitude of the unstable limit cycle seen with simulations in the previous sections, referring both to the simple car/simple model and complex car/complex model.

Note that the ability of the human driver to adapt to the manoeuvre allows, after a transient, the red curve to come back to the desired equilibrium state. From the tests at the simulator we argue that an understeering vehicle driven by a human is not always stable, but its dynamical response depends on the magnitude of the perturbation.

The second vehicle configuration is oversteering. To estimate the bifurcation speed, the longitudinal speed has been increased from $15 \mathrm{~m} / \mathrm{s}$, with low longitudinal acceleration in order to 


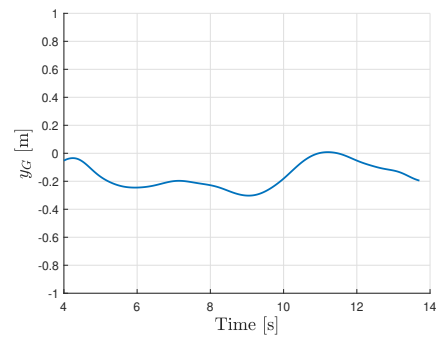

(a) Lateral position

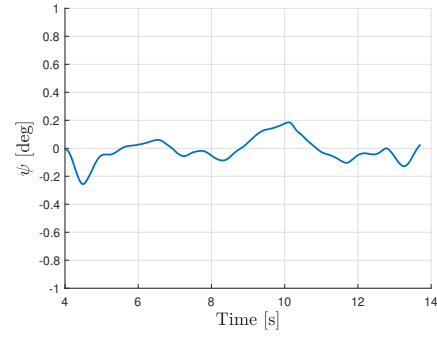

(b) Yaw angle

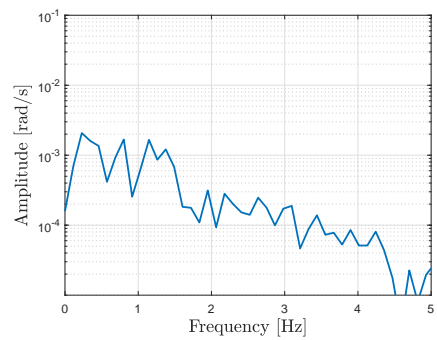

(d) Yaw rate FFT

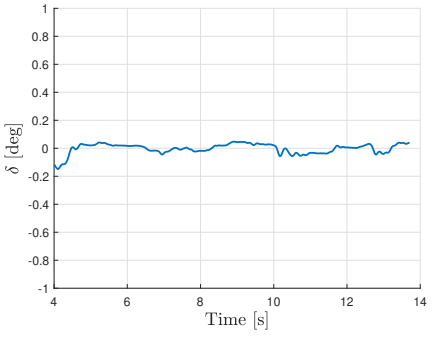

(c) Steering angle

Figure 19: Experimental results. Understeering configuration. Chaotic motion, $u=90 \mathrm{~m} / \mathrm{s}$. Data in Tab.4.

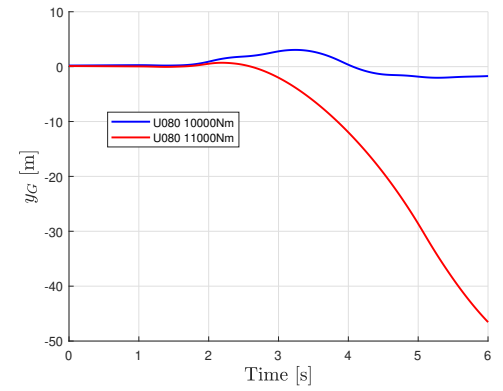

(a) Lateral position

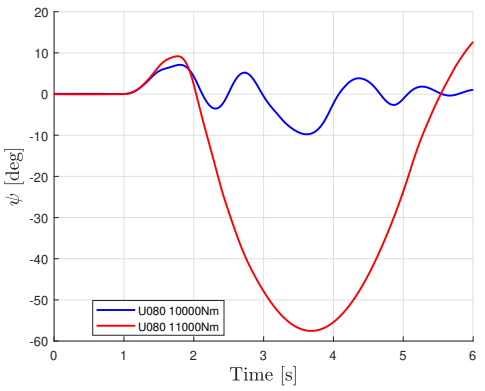

(b) Yaw angle

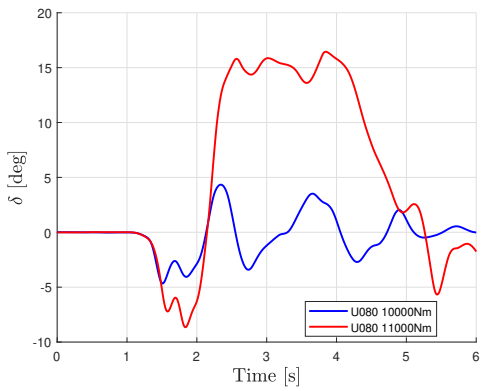

(c) Steering angle

Figure 20: Experimental results. Understeering configuration. A slight increase of disturbance (second perturbation strategy) causes instability, $u=80 \mathrm{~m} / \mathrm{s}$. Data in Tab.1 and Tab.4.

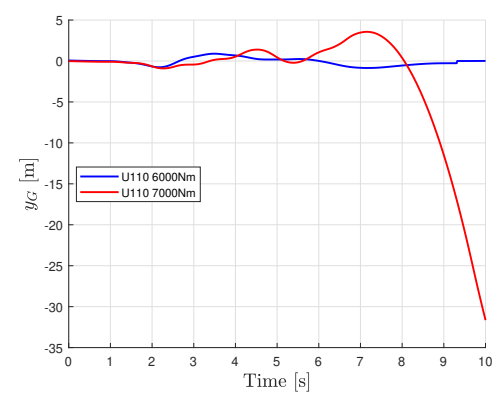

(a) Lateral position

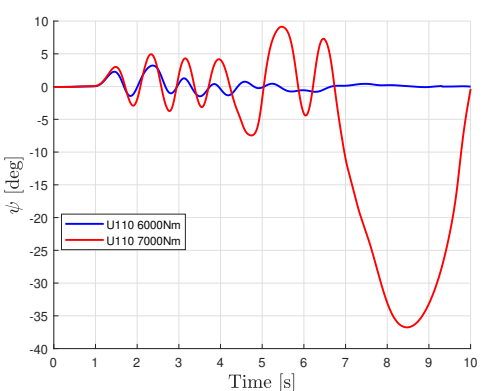

(b) Yaw angle

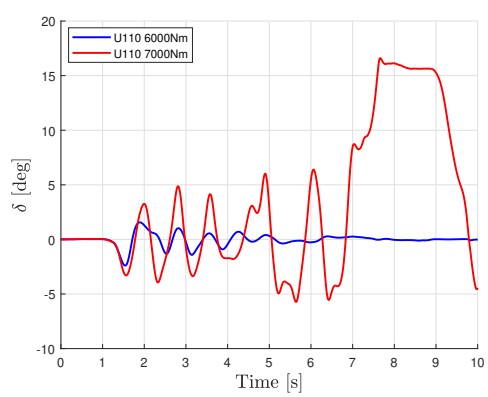

(c) Steering angle

Figure 21: Experimental results. Understeering configuration. A slight increase of disturbance (second perturbation strategy) causes instability, $u=110 \mathrm{~m} / \mathrm{s}$. Data in Tab.1 and Tab.4. 


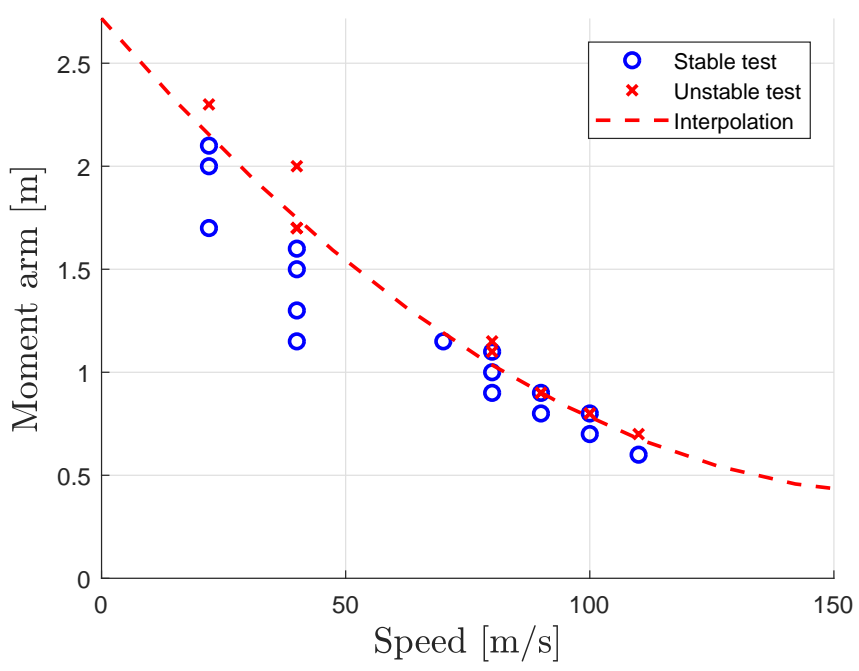

Figure 22: Experimental results. Understeering configuration. Estimation of the unstable limit cycle amplitude. Data in Tab.1 and Tab.4

make negligible the longitudinal tire forces. The results are reported in Fig. 23, where the longitudinal speed time history is presented. The dynamical response of the system can be divided in two kinds, one characterizing longitudinal speeds lower than $50 \mathrm{~m} / \mathrm{s}$ and the second for higher speeds. The first dynamical response is similar to the low amplitude chaotic motion seen with the understeering configuration, possibly caused by road irregularities or by the driver. When the speed of $50 \mathrm{~m} / \mathrm{s}$ is passed the chaotic motion gets bigger amplitudes. The different types of motion can be seen by comparing the frequency spectra at low and at high forward velocity, reported in Fig. 23f. It is clear how the amplitudes of the frequency components increase in all the frequency domain when the speed is higher than $50 \mathrm{~m} / \mathrm{s}$. Analyzing the time response, beatments-like behaviour can be seen, caused by the presence of two main harmonic components with similar frequency, one at $0.38 \mathrm{~Hz}$ and the second at $0.42 \mathrm{~Hz}$. At $50 \mathrm{~m} / \mathrm{s}$ a supercritical Hopf bifurcation occurs. The human driver is able to control the vehicle after the bifurcation speed, although it is not able to stabilize the system to the follow a straight path, since the motion is chaotic.

In Fig. 24 the results of the experimental tests performed to investigate the presence of the unstable limit cycle are reported. It can be seen that also in this oversteering configuration an unstable limit cycle is always present. The amplitude of the cycle at the same speed compared to the one of the understeering configuration is lower. To make the system unstable with an oversteering car the magnitude of the perturbation that has to be provided is lower.

\section{Conclusion}

The paper deals with the safety of road vehicles during straight ahead running. Both an understeering and an oversteering car are considered. The study is both theoretical and experimental. Different mathematical models of cars and drivers are considered. The combination of a simple car model with a simple driver model allowed to establish a link between this paper with previous theoretical studies presented in the literature. We manged to confirm that bifurcations during straight ahead motion can be found. Such bifurcations are often of the Hopf kind. They are either sub- or, less frequently, super-critical. The existence of bifurcations, namely Hopf bifurcations, is found for both the simple car /simple driver model combination and for the complex car/ complex driver model combination. This qualitative correspondence seems an original contribu- 


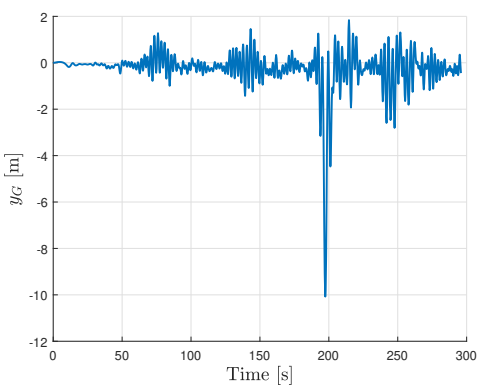

(a) Lateral position

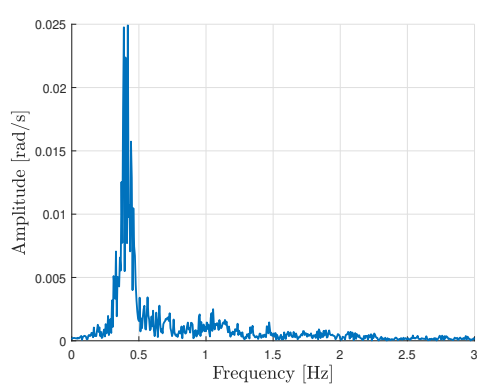

(d) Frequency spectrum: Yaw rate $(\mathrm{e}$ (0-170 s)

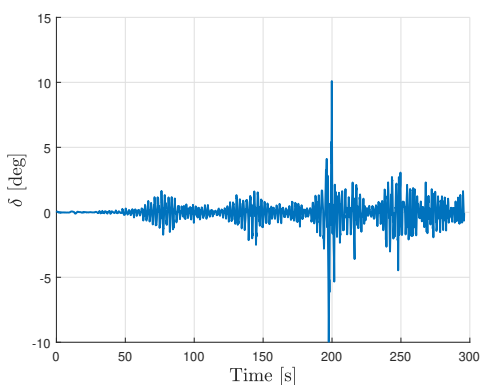

(b) Steering angle

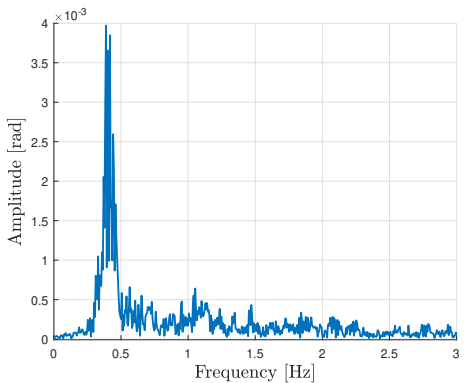

(e) Frequency spectrum: Steering angle $(0-170 \mathrm{~s})$

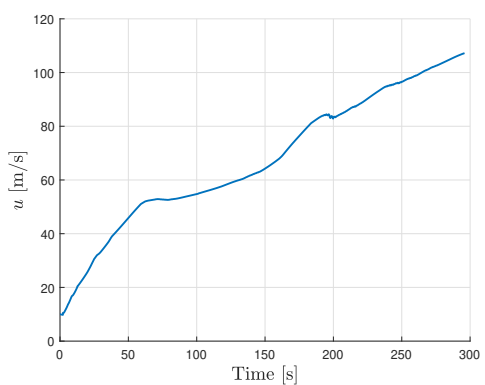

(c) Longitudinal speed

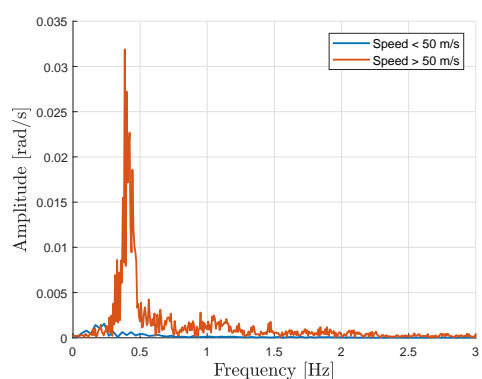

(f) Yaw rate frequency spectrum at low (blue) and high (red) speed.

Figure 23: Experimental tests. Oversteering configuration. Estimation of the bifurcation speed and evaluation of the chaotic motion after the bifurcation. Data in Tab.2 and Tab.4.

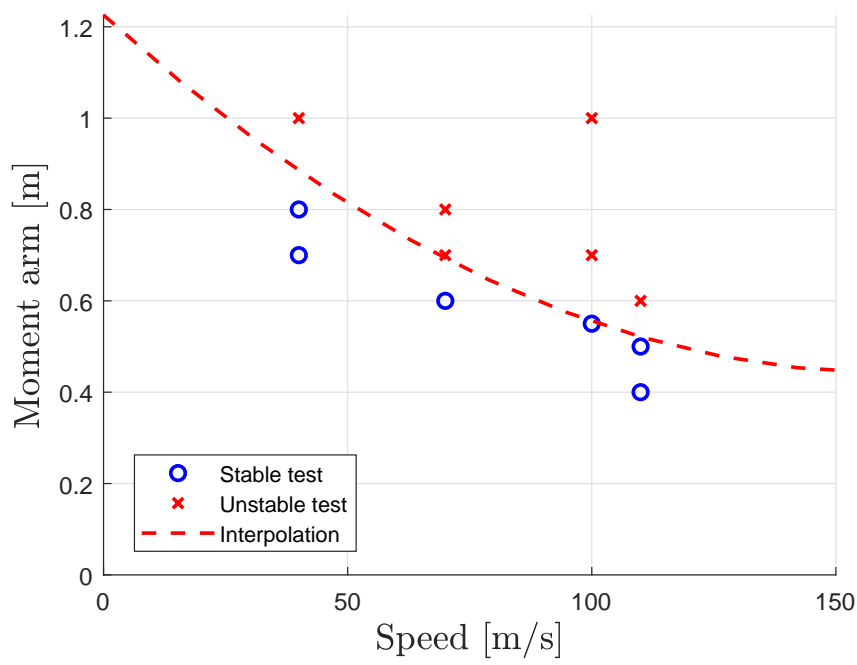

Figure 24: Experimental results. Oversteering configuration. Estimation of the unstable limit cycle amplitude. Data in Tab.2 and Tab.4. 
tion produced in the paper. Such a correspondence is extremely useful for interpretation of the simulations performed by a complex car/ complex driver model combination. The amplitudes of the limit cycles and the critical speeds are different between the simple car / simple driver model combination and for the complex car/ complex driver model combination. This, of course depends on both the car model and the driver model. With such a comparison we have somehow substantiated the previous papers in the literature that predicted bifurcations resorting to simple models only. The experimental study involved the employment of a driving simulator in which a human driver controlled the complex car model. The driving simulator does not seem introducing negative effects able to influence the occurrence of bifurcations, actually the latence of the simulator is more than one order of magnitude smaller than the driver's time lag. Tests at the simulator seem to be the only possible way to study experimentally the running safety of cars. At the driving simulator bifurcations were found which correspond qualitatively to the ones predicted either with the simple car / simple driver model combination or the complex car / complex driver model combination. The correspondence that was found was reasonable but not quantitatively accurate. In fact, especially the simple driver model seems inadequate to capture the actual human driver behaviour. Additionally, the driver adapts to the car behaviour so some differences were found between subsequent runs. The theoretical prediction made in the literature with a simple car/simple driver model combination, referring to a chaotic motion occurring after a subcritical Hopf bifurcation, was experimentally substantiated at the driving simulator. The nature of bifurcations that have been found seem real, i.e. they could be found on an actual car running straight ahead. It is a common belief that understeering vehicles are (globally) stable independently from the driving velocity: our study shows that this is not the case when the driver is in the loop. This result is confirmed both by theoretical results (using either simple or complex models) and by experiments with the driving simulator. Additionally, in the oversteering case, at speeds higher than the critical one, the motion is heavily chaothic. The study has disclosed a wealth of possible behaviours of cars, all useful to provide designers information on proper design of chassis systems, namely: tyres, suspensions, steering, powertrain and, even, in general, vehicle architecture. The study will evolve in the future into considering more complex driving manoeuvres like running into a bend or lane change. The existence or not of subcritical Hopf bifurcations should be carefully investigated for automated or autonomous vehicles.

\section{References}

[1] Danisi engineering, via ippolito nievo 62, nichelino (to) - italy. www.danisieng.com.

[2] Vi-grade: Driving simulation - dynamic driving simulator. www.vi-grade.com/en/ products/vi-carrealtime/.

[3] C. Bobier-Tiu, C. Beal, J. Kegelman, R. Hindiyeh, and J. Gerdes. Vehicle control synthesis using phase portraits of planar dynamics. Vehicle System Dynamics, 2018. cited By 2; Article in Press.

[4] B. Catino, S. Santini, and M. Di Bernardo. Mcs adaptive control of vehicle dynamics: an application of bifurcation techniques to control system design. In 42nd IEEE International Conference on Decision and Control, volume 3, pages 2252-2257. IEEE, 2003.

[5] F. Della Rossa, M. Gobbi, G. Mastinu, C. Piccardi, and G. Previati. Bifurcation analysis of a car and driver model. Vehicle System Dynamics, 52(sup1):142-156, 2014.

[6] F. Della Rossa and G. Mastinu. Analysis of the lateral dynamics of a vehicle and driver model running straight ahead. Nonlinear Dynamics, 92(1):97-106, 2018. 
[7] F. Della Rossa and G. Mastinu. Straight ahead running of a nonlinear car and driver modelnew nonlinear behaviours highlighted. Vehicle system dynamics, 56(5):753-768, 2018.

[8] F. Della Rossa, G. Mastinu, and C. Piccardi. Bifurcation analysis of an automobile model negotiating a curve. Vehicle System Dynamics, 50(10):1539-1562, 2012.

[9] A. Dhooge, W. Govaerts, and Y. A. Kuznetsov. Matcont: a matlab package for numerical bifurcation analysis of odes. ACM Transactions on Mathematical Software (TOMS), 29(2):141-164, 2003.

[10] T. D. Gillespie. Fundamentals of vehicle dynamics. Technical report, SAE, 1992.

[11] J. Guckenheimer and P. Holmes. Nonlinear Oscillations, Dynamical Systems, and Bifurcations of Vector Fields. Springer, 1983.

[12] K. Guo and H. Guan. Modelling of driver/vehicle directional control system. Vehicle System Dynamics, 22(3-4):141-184, 1993.

[13] Z. Hao, L. Xian-sheng, S. Shu-ming, L. Hong-fei, G. Rachel, and L. Li. Phase plane analysis for vehicle handling and stability. International journal of computational intelligence Systems, 4(6):1179-1186, 2011.

[14] H. Hu, Z. Gao, Z. Yu, and Y. Sun. An experimental driving simulator study of unintentional lane departure. Advances in Mechanical Engineering, 9(10):1687814017726290, 2017.

[15] H. Hu and Z. Wu. Stability and hopf bifurcation of four-wheel-steering vehicles involving driver's delay. Nonlinear Dynamics, 22(4):361-374, 2000.

[16] Y. A. Kuznetsov. Elements of applied bifurcation theory, volume 112. Springer Science \& Business Media, 2013.

[17] D.-C. Liaw, H.-H. Chiang, and T.-T. Lee. Elucidating vehicle lateral dynamics using a bifurcation analysis. IEEE Transactions on Intelligent Transportation Systems, 8(2):195$207,2007$.

[18] Z. Liu and G. Payre. Global bifurcation analysis of a nonlinear road vehicle system. Journal of Computational and Nonlinear Dynamics, 2(4):308-315, 2007.

[19] Z. Liu, G. Payre, and P. Bourassa. Nonlinear oscillations and chaotic motions in a road vehicle system with driver steering control. Nonlinear Dynamics, 9(3):281-304, 1996.

[20] Z. Liu, G. Payre, and P. Bourassa. Stability and oscillations in a time-delayed vehicle system with driver control. Nonlinear Dynamics, 35(2):159-173, 2004.

[21] C. C. Macadam. Understanding and modeling the human driver. Vehicle System Dynamics, 40(1-3):101-134, 2003.

[22] G. Mastinu, F. Della Rossa, and C. Piccardi. Nonlinear dynamics of a road vehicle running into a curve. In Applications of Chaos and Nonlinear Dynamics in Science and EngineeringVol. 2, pages 125-153. Springer, 2012.

[23] G. Mastinu, A. Lattuada, and G. Matrascia. Straight-ahead running of road vehicles-analytical formulae including full tyre characteristics. Vehicle System Dynamics, in press(-):-, 2018.

[24] G. Mastinu and M. Ploechl. Road and off-road vehicle system dynamics handbook. CRC press, 2014. 
[25] M. Mitschke and H. Wallentowitz. Dynamik der kraftfahrzeuge, volume 4. Springer, 1972.

[26] E. Ono, S. Hosoe, H. D. Tuan, and S. Doi. Bifurcation in vehicle dynamics and robust front wheel steering control. IEEE transactions on control systems technology, 6(3):412-420, 1998.

[27] H. Pacejka. Tire and vehicle dynamics. Elsevier, 2005.

[28] M. Plöchl and J. Edelmann. Driver models in automobile dynamics application. Vehicle System Dynamics, 45(7-8):699-741, 2007.

[29] G. Reymond, A. Kemeny, J. Droulez, and A. Berthoz. Role of lateral acceleration in curve driving: Driver model and experiments on a real vehicle and a driving simulator. Human factors, 43(3):483-495, 2001.

[30] S. Shen, J. Wang, P. Shi, and G. Premier. Nonlinear dynamics and stability analysis of vehicle plane motions. Vehicle System Dynamics, 45(1):15-35, 2007.

[31] S. Shi, L. Li, X. Wang, H. Liu, and Y. Wang. Analysis of the vehicle driving stability region based on the bifurcation of the driving torque and the steering angle. Proceedings of the Institution of Mechanical Engineers, Part D: Journal of Automobile Engineering, 231(7):984-998, 2017.

[32] S. H. Strogatz. Nonlinear Dynamics and Chaos with Student Solutions Manual: With Applications to Physics, Biology, Chemistry, and Engineering. CRC Press, 2018.

[33] S. Tousi, A. Bajaj, and W. Soedel. Finite disturbance directional stability of vehicles with human pilot considering nonlinear cornering behavior. Vehicle System Dynamics, 20(1):2155, 1991.

[34] H. True. On the theory of nonlinear dynamics and its applications in vehicle systems dynamics. Vehicle System Dynamics, 31(5-6):393-421, 1999.

[35] X. Wang and S. Shi. Analysis of vehicle steering and driving bifurcation characteristics. Mathematical Problems in Engineering, 2015, 2015.

[36] X. Wang, S. Shi, L. Liu, and L. Jin. Analysis of driving mode effect on vehicle stability. International journal of automotive technology, 14(3):363-373, 2013.

[37] D. H. Weir. Models for steering control of motor vehicles. In Proceedings, Fourth Annual NASA-University Conference on Manual Control, 1968, pages 135-169. US Government Printing Office, 1968. 